\title{
Measurements of TYVEK Reflective Properties for the Pierre Auger Project
}

\author{
By \\ Justus Ogwoka Gichaba
}

The University of Mississippi

August 1998 


\begin{abstract}
We have measured the spectrum and diffuse reflection of various samples of Tyvek, a material to be used to line the inner walls of the Pierre Auger Observatory water cerenkov tanks. These measurements were carried out with a Lambda 18 UV/VIS spectrometer over a wavelength range from $200 \mathrm{~nm}$ to $700 \mathrm{~nm}$. The angular dependance of this scattering was a gaussian. We have also carried the measurements with the PASCO OS-8020 to find the reflectivity of Tyvek samples versus Incident and Reflected angles. The reflected angles range from $-90^{\circ}$ to $-90^{\circ}$. Finally, information from these measurements was used to simulate Cosmic rays events in a Water Cerenkov detector.
\end{abstract}




\section{Preface}

The Pierre Auger Observatory will consist of a huge ground array of water cerenkov vessels working in unison with a fluorescent atmospheric detector. These detectors are being designed to measure the energy and direction of arrival of very high energy cosmic rays from unknown galactic or extra-galactic sources. Understanding of these $10^{20} \mathrm{eV}$ cosmic rays will help us understand the complex and profound workings of the cosmos. 


\section{Contents}

1 Introduction 1

1.1 Cosmic Ray Air Showers . . . . . . . . . . . . . . . . . . . . 3

1.1.1 Detecting air showers . . . . . . . . . . . . . . 4

1.1.2 The Shower, the Glow, and the Pancake . . . . . . 7

1.2 Water Cerenkov Detectors .............. . . 8

1.2.1 The photomultiplier Tube ............ . 10

1.2.2 Cerenkov Light Emitted by Relativistic Charged Particles .................... 12

1.2.3 Existing Water Cerenkov Detector . . . . . . . . . . 14

1.2.4 Pierre Auger Surface \& Fluorescence Detectors . . . . 16

2 Reflectivity Tests of Tyvek Samples 18

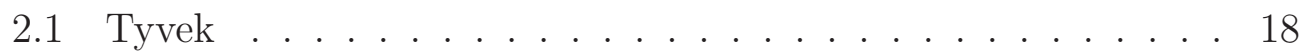

2.1.1 Specular \& Diffuse Reflection . . . . . . . . . . . . . 19

2.2 Measurement of Tyvek Samples . . . . . . . . . . . . . . . . 21

2.3 Perk \& Elmer Lambda-18 Spectrometer and Setup . . . . . . 22

2.4 Measurement Technique ... . . . . . . . . . . 23

2.5 Test Scans . . . . . . . . . . . . . . . . . 25

2.6 Corrections to the Data . . . . . . . . . . . . 30

2.7 Error Analysis . . . . . . . . . . . . . . . . . . 30

$3 \quad$ TYVEK Reflectivity versus Incident and Reflected Angles 32 


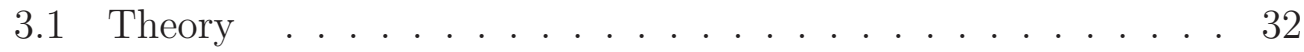

3.2 Experimental Setup and Procedure . . . . . . . . . . . 34

3.3 In-the-Plane Measurements . . . . . . . . . . . . . . . 35

3.4 Out-of-Plane Measurements . . . . . . . . . . . . . . . . . . . 39

4 Tank Simulation $\quad 40$

4.1 Measurements .................... . . 42

5 Conclusions 46 


\section{List of Figures}

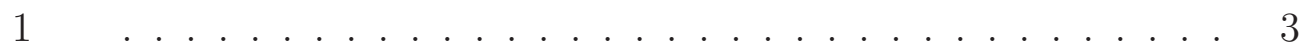

2 The Layout of Auger Observatory _ . . . . . . . . . . 6

3 PHOTOMULtiplieR TUBE . . . . . . . . . . . . 11

$4 \quad$ FLY'S EYE . . . . . . . . . . . . . . . . 17

5 Diffuse Reflection . . . . . . . . . . . . . 20

$6 \quad$ Specular \& Diffuse Reflection . . . . . . . . . . . . . . 21

7 Experiment Setup(Drawing) (Perk 63 Elmer -18 spectrometer) 23

8 Experiment Setup(Drawing) (Perk 63 Elmer -18 spectrometer) 23

9 The Reflectivity Tests . . . . . . . . . . . . . 26

10 The Reflectivity Tests . . . . . . . . . . . . . . 27

11 The Reflectivity Tests . . . . . . . . . . . . . 28

12 Corrected Specular \& Diffuse Histograph for the 12 Tyvek

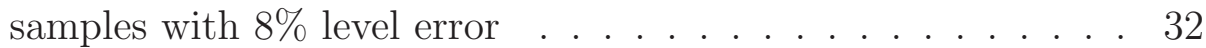

13 The Experiment Setup . . . . . . . . . . . . . . . . 35

14 Intensity of the reflected light in arbitrary units as a function of the reflected angle for incident angles $0^{\circ}, 10^{\circ}$, and $30^{\circ} \ldots 37$

15 The Reflectivity Tests . . . . . . . . . . . . . . 38

16 Reflectivity dependence of the wavelength and the Incident angle 41

17 Comparison of average pulses due to central vertical throughgoing muons with tyvek top and black lining on the top. . . . 45 


\section{Introduction}

Cosmic Rays are now known to span the energy range from $10^{9} \mathrm{eV}$ to beyond $10^{20} \mathrm{eV}[1]$. Above $10^{14} \mathrm{eV}$ the particles are so rare that their detection relies on measurements of the giant cascades or extensive air showers created in the atmosphere. The giant cascades or extensive air showers can be observed with arrays of particle and optical detectors at ground level. Since the flux of particles falls inversely as the square of the energy until above $10^{19} \mathrm{eV}$ only about one per $\mathrm{km}^{2}$ per year is collected.

The existence of the most energetic event yet detected, $3 \times 10^{20} \mathrm{eV}$, implies that it must have originated within $20 \mathrm{Mpc}$ of the earth[2]. However the arrival directions of this event and others of similar energy do not point to any unusually energetic objects within our galaxy or elsewhere. The origin of these very high energy particles is unknown and how Nature can accelerate them to energies is not understood.

Only a few hundred records above $10^{19} \mathrm{eV}[3]$ have been recorded thus far, too few to draw major conclusions. Increasing this sample will require a much greater effort. The Pierre Auger Project is a proposal to construct a device with an aperture of $5000 \mathrm{~km}^{2}$ sr per year. Such an instrument would allow 5000 events to be recorded annually above $10^{19} \mathrm{eV}$ and, with the capability of accurate energy and primary mass determinations, and will provide data to confront conflicting ideas about the origin of the most energetic particles in nature. 
Two giant observatories will consist of a large array of water Cerenkov detector tanks, shown in Fig 1, spaced apart over a $5000 \mathrm{~km}^{2}$ area. In these facilities extremely high-energy cosmic rays will be detected through the Cerenkov effect produced in the water by the associated air shower secondary particles entering the tanks. If this project is successful, an accurate, highstatistics measurement of the energy spectrum, arrival directions, and nuclear identity of the highest energy cosmic rays will be made, possibly answering many questions on the nature and the origin of these high energy cosmic events.

This work focuses on the design of the large water Cerenkov tanks used to detect cosmic rays at ground level. Quantitative information referent to different air shower parameters is expected to be obtained by measuring the Cerenkov light intensity for each event. For this reason it is very important to assure that the sensitive photodetectors (photomultiplier tubes) will collect as much as possible of the generated light. The two main properties of these detectors which control the light collection efficiency are (1) the attenuation length of the water as function of the wavelength $(\lambda)$ and (2) the reflectivity of the inner liner (eg., Tyvek) as a function of wavelength $(\lambda)$ and the incident angle [6]. Both issues are currently under study, and the second a subject of this work. In addition we have conducted a simulation of the performance of a water cerenkov detector (TANK) a model based on the proposed surface array detectors for the Auger Observatory. 


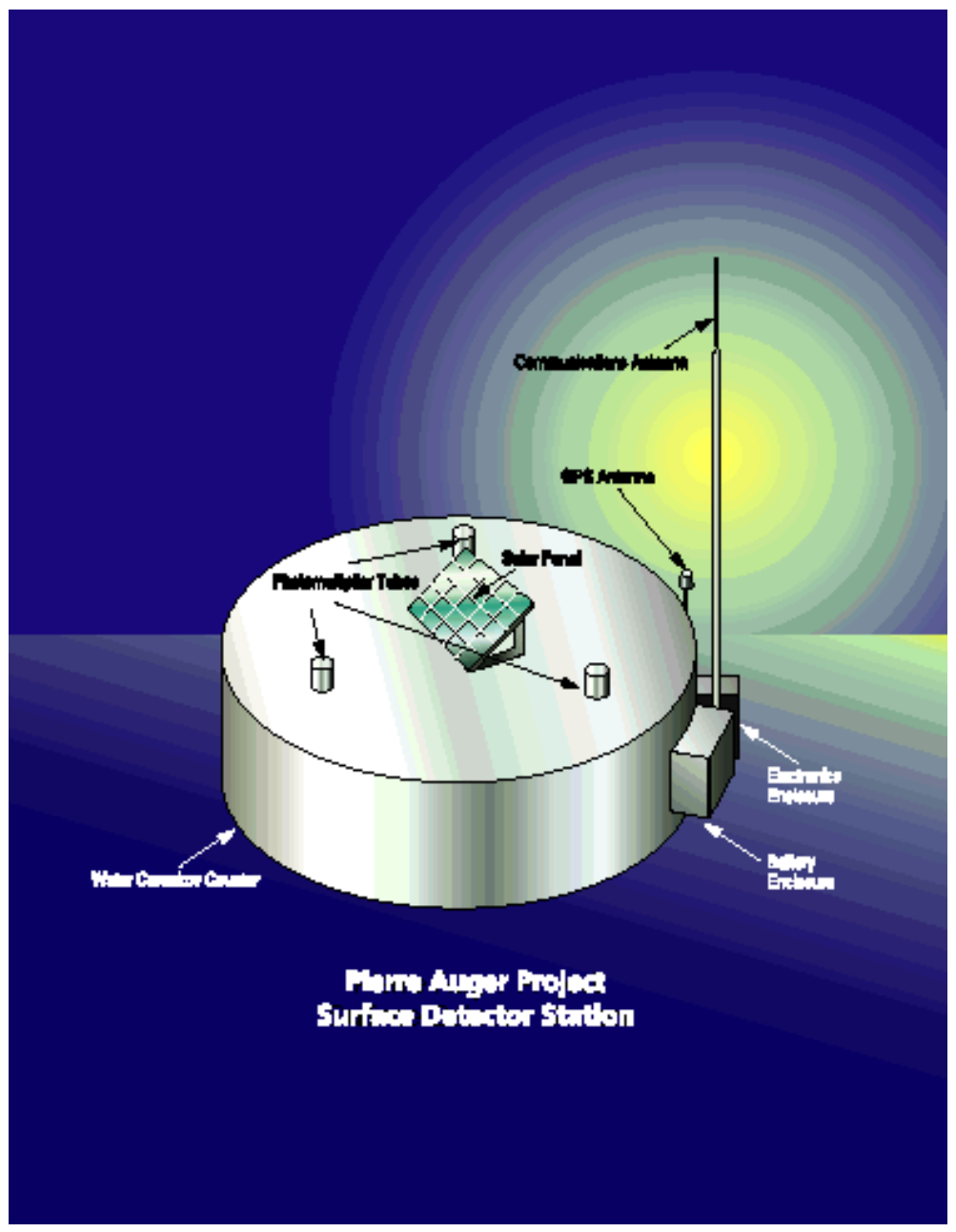

Figure 1: The Surface Detector

\subsection{Cosmic Ray Air Showers}

Cosmic rays are sub-atomic particles and gamma-ray photons which bombard the Earth from outer space. They possess a large range of energies (usually measured in electron-volts $[\mathrm{eV}])$ from a few $\mathrm{GeV}$ to more than $100 \mathrm{EeV}$.

Low energy cosmic rays are most plentiful many thousand per square $\mathrm{m}$ 
per second. The highest energy cosmic rays are very rare, less than one per square $\mathrm{km}$ per century. This makes compositional studies at high energies very difficult. Usually the vast majority of cosmic rays are single protons, although other heavier atomic nuclei are also present in the primary cosmic ray flux, extending all the way up to iron nuclei. The vast majority of primary cosmic ray particles are therefore positively charged.

A small fraction $(0.1 \%)$ of cosmic rays are photons in the form of gammarays. These gamma-ray photons are important when trying to find the origin of cosmic rays since they are uncharged and arrive at the Earth undeflected by the galactic magnetic field.

In general the highest energy cosmic rays are very great interest to the scientific community, providing a unique tool for locating the high energy sources. Just as with gamma-rays the charged component is relatively undeflected by the galactic magnetic fields and therefore arrive at the earth on a true course. The arrival directions should theoretically be very close to the direction of the source in the sky.

\subsubsection{Detecting air showers}

Direct observation of cosmic rays is possible only above the earth's atmosphere. High energy cosmic rays are so rare though, that it would be impossible to lift into space a detector big enough to capture a significant number of them, so one has to find ways to detect them at the surface. When cosmic ray particles strike the atmosphere, collisions with air molecules initiate 
cascades of secondary particles. These "air showers" often containing many millions of particles, cascade to earth over a large lateral area. When they reach the surface of the earth, the particles from an air shower initiated by a $10^{20} \mathrm{eV}$ cosmic ray may cover an area of 16 square kilometers.

The atmosphere absorbs the great energy of the incident (primary) cosmic ray particles in a giant air shower which develops after an initial interaction with an atmospheric gas molecule. Thus nature provides us with most of the experimental apparatus in the form of the atmosphere needed to detect cosmic rays. This showering process makes it possible to detect and measure the properties of the primary at ground level. By careful measurement of the air shower one can estimate the direction and energy of the original incoming cosmic ray particle.

Cosmic rays are detected in a variety of ways depending on their energy. (1) The lowest energy cosmic rays are detected by equipment on board satellites and high altitude balloons because they are absorbed readily in upper atmosphere. (2) Higher energy cosmic rays interact in the atmosphere producing cosmic ray air showers. Many of the air showers are absorbed in the atmosphere and do not reach ground level. However, the air shower particles initially travel at speed greater than that of light in air and emit tiny flushes of light (Cerenkov light). The Cerenkov light can be detected at ground level using air-cerenkov detectors on clear, dark, moonless nights. (3) Even higher energy cosmic rays produce air showers which reach the ground level and can be detected directly with particle detectors. The detectors are usually 
arranged in a grid formation( or array), Figure 2, on the ground and the arrival direction of the initial cosmic ray primary particle can be inferred by measuring the particle arrival times at each of the detectors.

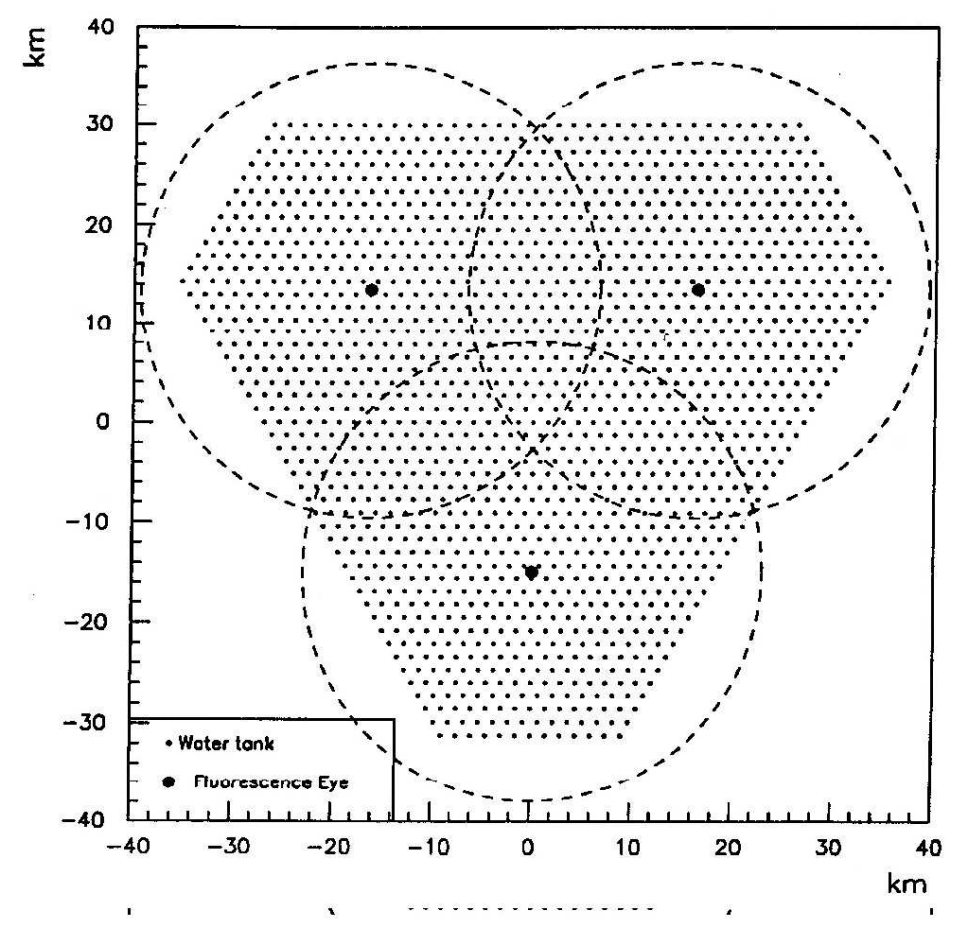

Figure 2: The Layout of Auger Observatory

Within a water Cerenkov detector of such a ground array the cerenkov light will be produced with a distinct $1 / \lambda^{2}$ spectrum. A bialkai photocathode and appropriate UV transmitting window will be sensitive to light $\lambda=(300-$ $600) \mathrm{nm}$. Since the cerenkov light spectrum is weighted heavily in the UV, a 
long absorption length in the water will be crucial.

\subsubsection{The Shower, the Glow, and the Pancake}

When a high-energy cosmic ray enters the earth's atmosphere it interacts and creates a shower of lower-energy particles. These particles in turn create more particles. The net result is a large pancake of lower energy particles (100's to 1000's) traveling toward the earth at approximately the speed of light. This pancake is roughly 100 meters across and 1-3 meters wide. The particles in the pancake are photons, electrons, and positrons (the electron's antimatter partner). If the initial cosmic ray was a nucleus (i.e. Hydrogen, Helium, Iron, etc.) there will also be muons in the showers. Muons are heavy electrons that have great penetrating power[7].

As the electrons and positrons travel through the atmosphere they emit Cerenkov light. That is because the are traveling faster than the speed of light in air. If the cosmic ray that started the shower of particles does not have sufficient energy then very few of the secondary particles (0 - 100's) will reach the ground. But the Cerenkov light they generated in the atmosphere will.

This Cerenkov light also arrives in a pancake, but now the pancake is about 200 meters across and again only 1-3 meters thick. Given the speed of light, that means that all of the Cerenkov light arrives in about 10 nanoseconds ( 1 nanosecond $=1$ billionth of a second). Using large mirror arrays the Cerenkov light can be focused onto photomultiplier tubes[8]. By requiring a 
fast coincidence of many photons coming within a few 10's of nanoseconds, the pancake can be detected.

The few secondary particles that reach the ground requires a large and well designed detectors to detect them. They a few already prototypes of detectors that are in operation and have proven well to be effective. The water Cerenkov detectors are the primary detectors chosen for these project.

\subsection{Water Cerenkov Detectors}

Many types of detectors were considered for use in the Auger surface array. Water-Cerenkov tanks, when compared to other technologies such as plastic scintillators, are a good choice as the the surface array detector for several reasons. One being that it is a proven technology. For twenty years a 12 $\mathrm{km}^{2}$ air shower array employing more than 200 water-cerenkov units was operated at Haverah Park in the UK [9]. The experience gained during that experiment provides a much useful information for the Auger project, demonstrating that an array based on this technique can operate for along period with both stability and low maintenance. This being the primary concern for the Auger project which has a large number of stations over a very large area makes water cerenkov detectors[10] the primary detector technology for the Auger ground array. A suitably designed water tank array can adequately address the physics requirements, at the same time providing more cost effective than other techniques.

Water-Cerenkov tanks are especially sensitive to muons in air showers. 
The average muon energy is about two orders of magnitude higher than that of electrons or photons at ground level. Muons typically traverse the entire tank whereas most electrons and photons stop in water after penetrating a few centimeters.

This special sensitivity to muons is helpful to the goals of the Auger Project in a number of ways. One involves the study of cosmic ray composition. The number of muons in an air shower and their lateral extent at ground level is a powerful indicator of the nuclear identity of the of the primary cosmic rays. The lateral distribution of particles can be well determined by an array of this detector, enabling good estimation of the total size of the shower at the ground, and hence the primary energy. Muons tend to have larger transverse momenta with respect to the shower axis than electromagnetic particles because of the kinematics of their production is hadronic rather than electromagnetic in origin.

Proton air showers will be rich in muons and have large lateral extent due to the violence of the initial interaction. Showers from nuclear primaries will be muon rich and have lesser lateral extent. Gamma ray primaries will produce an electron rich shower and also be narrow at ground level. WaterCerenkov tanks permit good identification of the relative number of muons and electrons by measuring the amount of Cerenkov light produced by a particle traversing a tank as well as the time structure of such an event. Light from muon traversals will arrive at the photodetector slightly ahead of an electron signal loosing most of its energy near the top of the tan. We 
have studied the time structure of these processes in a water tank simulation in Section 4.

The Water Cerenkov detector can be simply be described as a volume of water acting as a Cerenkov radiator viewed by one or more sensitive light detectors, usually photomultiplier tubes.

\subsubsection{The photomultiplier Tube}

The photomultiplier has a light sensitive electrode called the photocathode which is placed on the inner surface of the glass which emits electrons when struck by photons. Inside the photomultiplier there is a vacuum. The electron is attracted and accelerated to the first dynode, which is charged positively by a high voltage. As it hits it with great energy, the dynode emits several electrons, which are then attracted to a second dynode, which has even higher positive electric potential. This process repeats many times. At the last dynode we have a large number of electrons. In this way the signal of a single electron is enormously amplified.

A photomultiplier schematic is shown in Fig 3, and a brief description of it's components is given below.

Photocathode Made of photosensitive material

Electrical optimal system Acts like electron collection system.

Anode The final signal is taken from here.

Multiplier It amplifies the weak primary photocurrent by using a series of secondary emission electrodes or DYNODES to produce a measurable current 
at the Anode of the Photomultiplier.

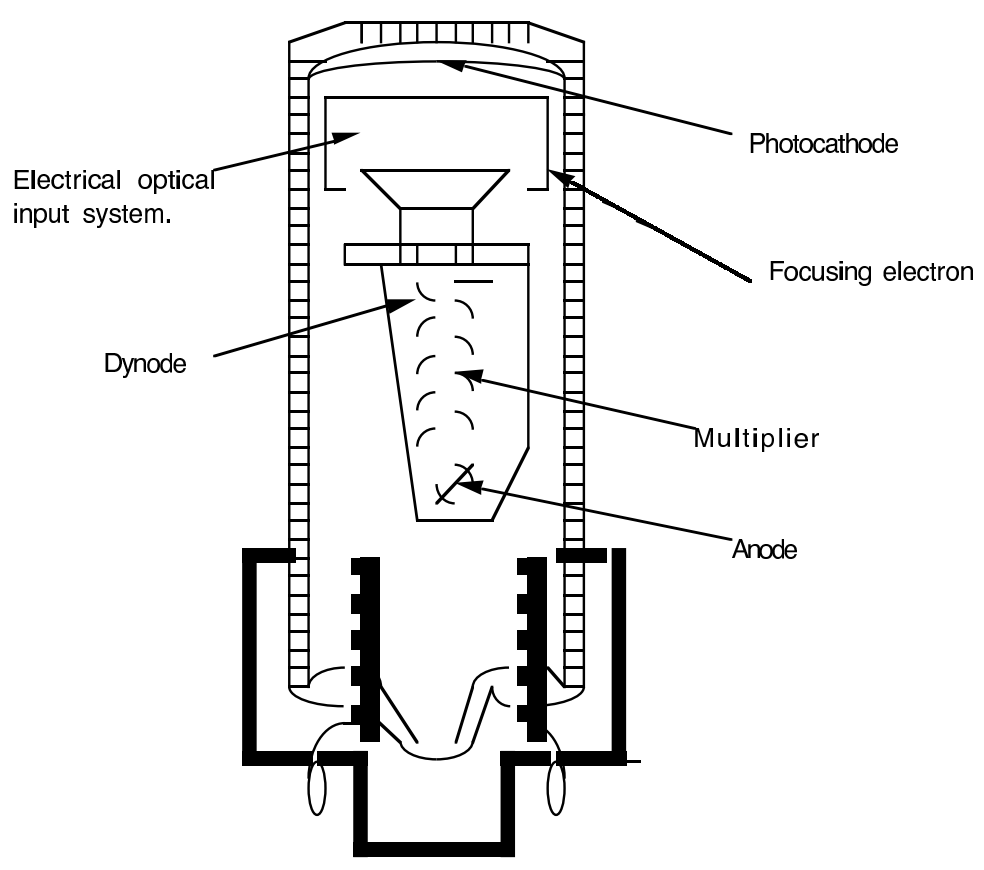

Figure 3: PHOTOMULTIPLIER TUBE 


\subsubsection{Cerenkov Light Emitted by Relativistic Charged Particles}

Cherenkov radiation is the radiation produced when a charged particle moves through a material at speed greater than the speed of light in that material. In simple dielectric material, the speed of light is given by [11]

$$
c_{n}=c / n .
$$

It is less than the speed of light in free space by a factor $1 / \mathrm{n}$, where $\mathrm{n}$ is the index of refraction for the material. Thus it's possible for a charge moving in a material to have a speed $\mathrm{v}$ that is greater than the speed of light in the material and still not violate Einstein's relativity. Upon exceeding the speed of light in the dielectric medium (eg., water) a burst of Cerenkov radiation is emitted along a direction making an angle $\theta_{c}$ with respect to the particle's direction of flight.

They are several distinctive properties of this radiation.

- Cherenkov radiation is asymmetric, being larger in the direction of motion of the particle and emitted with angle $\theta_{c}$ with respect to the particles direction of travel.

- Cherenkov radiation is linearly polarized, with the component of the electric field parallel to the path of the particles.

- The visible spectrum of Cherenkov radiation is continuos, with an energy varying as $\frac{d \lambda}{\lambda^{2}}$. 
The half-angle $\theta_{c}$ of the Cerenkov cone for a particle with velocity $\beta c$ in a medium with the index of refraction $n$ is

$$
\begin{gathered}
\theta_{c}=\cos ^{-1}\left(\frac{1}{n \beta}\right) \\
\theta_{c} \approx \sqrt{2\left(1-\frac{1}{n \beta}\right)} \quad\left(\text { for small } \theta_{c}\right)
\end{gathered}
$$

The threshold velocity $\beta_{t}$ is $\frac{1}{n}$, and

$$
\begin{aligned}
\gamma_{t} & =\frac{1}{\sqrt{1-\beta_{t}^{2}}} . \\
\text { Therefore, } \quad \beta_{t} \gamma_{t} & =\frac{1}{\sqrt{2 \delta+\delta^{2}}}, \text { where } \delta=n-1 .
\end{aligned}
$$

Cerenkov detectors [12] utilize one or more of the properties of Cerenkov radiation: (1) Their existences a momentum threshold $p_{t}=m_{o} \beta_{t} \gamma_{t}$ for emission of radiation. (2) The dependance of the Cerenkov cone half-angle $\theta_{c}$ on the velocity of the particle can be used to detect a particles identity. (3) The dependence of the number of emitted photons, on the particle's velocity can also be used to identify particle species.

The Pierre Auger Water-Cerenkov will identify particles based on the number of photons emitted in the water tanks as well as the timing information determined from the arrival of this light at the photodetector. The number of photoelectrons detected in a given device is a convolution of the number of Cerenkov photons produced in the radiator (water) of index of refraction $n$, length $L$, geometric collection efficiency $\epsilon_{\text {coll }}$ and whose photodetector converts photons to electrons with quantum efficiency $Q(\lambda)$ or $Q(E)$. This integral is shown below: 


$$
N_{\text {p.e. }}=370(e V-c m)^{-1} L \int_{E 1}^{E 2} \sin ^{2} \theta_{c} \epsilon_{\text {det }}(E) Q(E) d E
$$

The length of the radiator $L$ for vertical muons in the Pierre Auger water tank is $120 \mathrm{~cm}$. This and the angle of incidence will determine the number of photons generated along the cerenkov path, but a rough calculation indicates about 46000 photons emitted in $120 \mathrm{~cm}$ of track in the wavelength range $\lambda=(300-700) n m$. The light collection efficiency $\epsilon_{\text {det }}$ will depend on the number and quality of the geometrical reflections and the absorption length $(\Lambda \approx 7 m)$ for Cerenkov light in the water as each Cerenkov photon is moves to a photodetector. Once reaching the photodetector the efficiency for collecting the Cerenkov light $Q(\lambda)$ is the quantum efficiency of the photomultiplier tube for converting photons to an electrical signal $(\approx(10-20) \%)$. All of these properties are wavelength or energy dependent. We have studied this complex scenario of generation and collection of photons in our tank simulation.

\subsubsection{Existing Water Cerenkov Detector}

Super-Kamiokande [13] is a 50,000 ton ring-imaging water Cerenkov detector located at a depth of 2700 meters water equivalent in the Kamioka Mozumi mine in Japan. It is used mostly for the search for proton decay (nucleon decay in general), observation of neutrinos (solar, atmospheric, from muons created by cosmic ray particles in the atmosphere, and upward going muons created by neutrino interaction in the Earth beneath the detector). 
The detector consists of large tank of very clear water surrounded by a large array of photodetectors. The tank is a cylinder of roughly $40 \mathrm{~m}$ diameter and $40 \mathrm{~m}$ height. On all the walls (side, top and bottom) there are many (about 13000) photomultiplier tubes. They are especially light sensitive detectors that can detect single photons. They are 'looking' toward the inner volume of water. The walls of the Super-Kamionde tank are lined with Dupont Tyvek 1073-B to increase light collection efficiency.

To eliminate the background usually from the radioactivity in the surrounding rocks and water and to some extent cosmic muons. The detector is located deep underground in order to shield it from cosmic ray muon above it.

Milagro [14] consists of a 5 million gallon pool of water: 80 meters by 70 meters across and 8 meters deep. When the high energy secondary particles (electrons and positrons) travel through the water they emit Cerenkov light. (When a high energy photon enters the water it interacts and creates electrons and positrons.) This light spreads out in a 41 degree cone. An array of light sensitive detectors (photomultiplier tubes - PMTs) detects this light. Milagro will have 3 layers of PMTs. The top layer will have 450 PMTs on a 3 meter x 3 meter grid. These will float about 1-2 meters below the surface of the water. 


\subsubsection{Pierre Auger Surface \& Fluorescence Detectors}

The Auger Project will require detector units which will withstand a harsh outdoor desert environment for 20 years with very little maintenance.They must withstand high winds, hail,temperature extremes, and casual vandalism. An important step in the project is to develop a detector which meets these durability requirements as well as the performance requirements at a

cheaper cost. Several prototypes have been developed and more are still in construction. All of the prototype tanks have performed well as cosmic ray detectors.

The Auger Observatory layout shown in Figure 2 is to be a hybrid detector, employing two complementary techniques to observe extensive air showers. A giant array of particle counters will measure the lateral and temporal distribution of shower particles at ground level. An optical air-fluorescence detector will measure the air shower longitudinal development in the atmosphere above the surface array. Measurement of atmospheric fluorescence is possible only on clear, dark nights. About $15 \%$ of all Auger showers will be measured by both techniques.

The arrangement shown in Figure 2 is one of two separate, identical installations to be located in the northern and southern hemispheres respectively. In order to significantly increase the data set at the highest energies, Auger is a very large detector. The hybrid nature of the design enables accurate and robust determination of the energy and primary mass groups. Operating together, the surface array and fluorescence detector characterize showers to 
a greater degree than either technique alone.

Both the surface array and fluorescence methods are well established by prior experiments. The surface array resembles the array successfully employed by the Haverah Park group for over twenty years, although on a much larger scale. The fluorescence telescope uses techniques pioneered by the University of Utah's Fly's Eye [15], Figure 4, and the HiRes experiment[16].

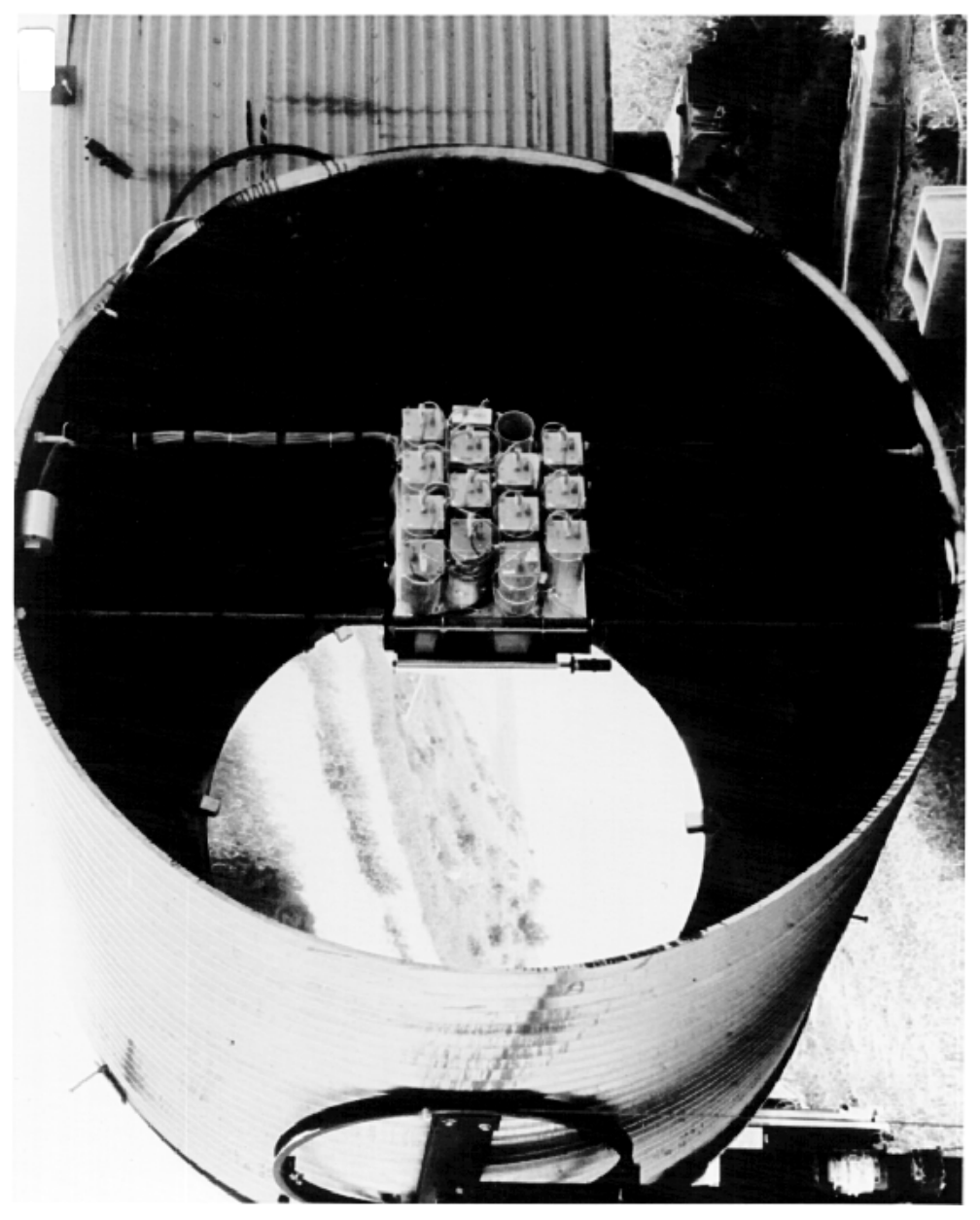

Figure 4: FLY'S EYE 
Table 1: Different Types of Tyvek Materials

\begin{tabular}{|c|c|c|}
\hline Sample & Type & Characteristics \\
\hline $1 \mathrm{~A}$ & $1025 \mathrm{D}$ & thickness $=5 \mathrm{mil}$ \\
\hline 3 & $1025 \mathrm{D}$ & thickness $=5 \mathrm{mil}$ \\
\hline 4 & $1056 \mathrm{D}$ & thickness $=5 \mathrm{mil}$ \\
\hline 5 & $1058 \mathrm{D}$ & thickness $=5 \mathrm{mil}$ \\
\hline 6 & $1059 \mathrm{~B}$ & thickness $=6 \mathrm{mil}$ \\
\hline 7 & $1059 \mathrm{D}$ & thickness $=8 \mathrm{mil}$ \\
\hline 8 & $1070 \mathrm{D}$ & thickness $=7 \mathrm{mil}$ \\
\hline 9 & $1073 \mathrm{~B}$ & thickness $=7 \mathrm{mil}$ \\
\hline 10 & $1073 \mathrm{D}$ & thickness $=8 \mathrm{mil}$ \\
\hline 11 & 1079 & thickness $=6 \mathrm{mil}$ \\
\hline 12 & $1085 \mathrm{D}$ & thickness $=10 \mathrm{mil}$ \\
\hline 15 & SR-1 & thickness $=10$ mil, coated at the back \\
\hline 16 & SR-2 & thickness $=14$ mil, coated at the back \\
\hline 17 & SR-3 & thickness $=14$ mil, coated at the back \\
\hline 18 & SR-4 & thickness $=16$ mil, coated at the back \\
\hline
\end{tabular}

\section{Reflectivity Tests of Tyvek Samples}

\section{$2.1 \quad$ Tyvek}

The material selected for the inner lining of the Pierre Auger [5] water tanks should have both highly diffuse and specular reflective properties. The Dupont material, Tyvek[4] has been proposed for this inner lining. A number of grades of Tyvek and it's laminates are manufactured. In this section we discuss our measurements of the reflectivity of DUPONT TYVEK samples as a function of wavelength. Because of its good reflectivity in the near $U V$ and durability, this material has been chosen to line the water tanks in the Pierre Auger Project ground array. Studies have shown TYVEK 1073B is 
approximately $90 \%$ reflective in the visible, dropping by a few percent as the angle of incidence increases. This studies also show that the reflectivity drops by (10-20)\% in the near UV [17].

In studying the reflective properties of Tyvek we are also exploring a technique that might be useful to the AUGER project for maintaining quality control in the manufacturing of many thousands ground array detectors. Our measurements are of a relative nature and taken on dry samples, but we feel they have been a useful guide for the choice of bag material in the water cerenkov detectors.

\subsubsection{Specular \& Diffuse Reflection}

Specular reflection is a reflection usually from a smooth surface. the bouncing of light rays from a surface in such way that the angle at which it strikes a given a ray is returned is equal to the angle at which it strikes the surface.

Diffuse reflection also known as Lambertian reflection [18] is a situation that arises when light is incident on a rough surface, it is reflected in many directions. The light is isotropically reflected with equal intensity in all directions. (see Figs 5,6 below)

For any given surface, the intensity only depends on the angle $\theta$ between the direction $L$ to the light source and the surface normal $N$ of of Figure 5b. They are two main reasons this occurs. First, Figure 5a shows a beam that intercepts a surface covers an area whose size is inversely proportional to the cosine of the angle $\theta$ that the beam makes with $N$. If the beam has 
an infinitesimally small cross-sectional differential area $d A$, then the beam intercepts an area $d A / \cos \theta$. This is the case for any surfaces regardless of the material.

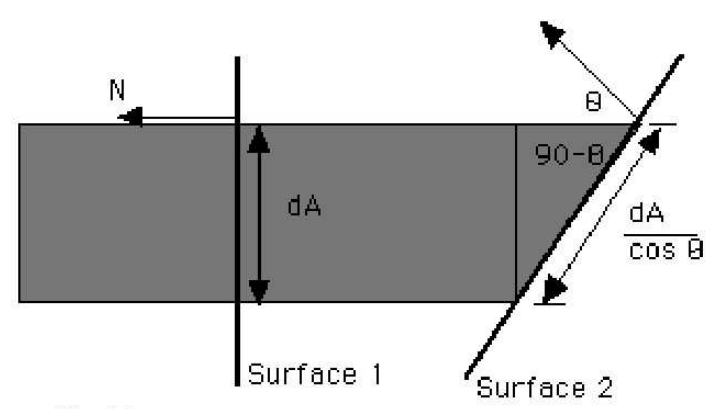

Fig 5 a.

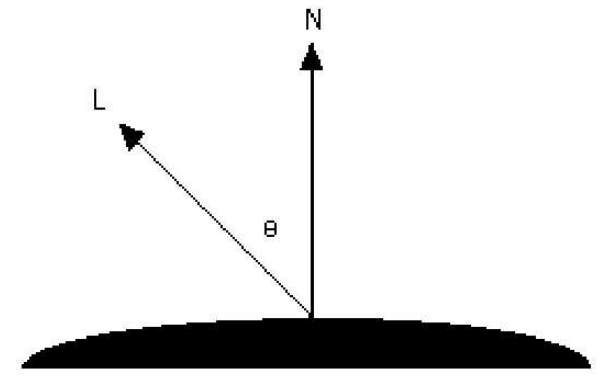

Fig $5 b$

Figure 5: Diffuse Reflection

Reflections from Lambertian surfaces have the property, often known as Lambert's Law[18], that the amount of light reflected from a unit differential area $d A$ toward a photometer is directly proportional to the cosine of the angle between the direction to the photometer and $N$. Since the amount of surface the in the photometer view is inversely proportional to the cosine of this angle, these two factors cancel out. Thus, for Lambertian surfaces, the 
amount of light detected is independent of the photometer direction and is proportional only to $\cos \theta$, the angle of incidence of the light.

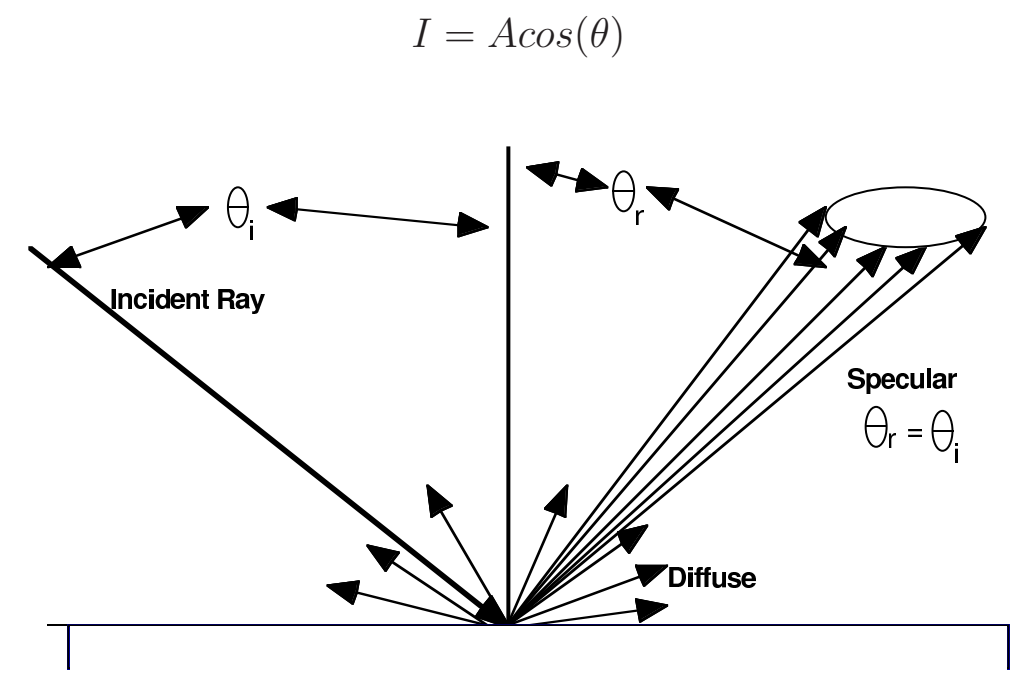

Figure 6: Specular \& Diffuse Reflection

\subsection{Measurement of Tyvek Samples}

In our studies, various samples of Tyvek were measured for the specular and diffuse reflective properties. Tyvek is a spun-woven polyolefin and there is no compelling reason to believe it has a uniform reflective properties with angle. The reflectance variation associated with the local weave pattern of the samples can dominate statistical variation. In addition, the roll to roll variations can be expected, probably at a level of (10-15)\%. Changes can also occur if the Tyvek is pressed to a backing material. For a true measurement of specular and diffuse reflectivity an integrating sphere technique should be employed with the final laminated samples. 
Such a study[17] has been performed for the super Kamiokande experiment, indicating a 90\% reflectivity for TYVEK 1073B, used as a reflector in their large water Cerenkov tank. Such a costly study was not possible for this work. We will measure the reflectance of different samples Tyvek as a function of wavelength with a relatively small solid angle. The spectrum shape in the near UV is most important to observe. An overall relative reflectivity for different sample is reported. A sample-to-sample variation is observed and described later.

\subsection{Perk \& Elmer Lambda-18 Spectrometer and Setup}

We have used the Perk \& Elmer lambda-18 spectrometer as the primary instrument in finding the reflectivity of Dupont Tyvek samples as a function of wavelength. The set up and sample placement in the spectrometer is shown in Figs 7 and 8. The scans were performed for a wavelength range $\lambda=200-$ $600 \mathrm{~nm}$. We have taken data for diffuse and specular reflections by switching between the two different sample placement positions in the spectrometer. For specular measurements the sample is placed to such that $\theta_{\text {incident }}=$ $\theta_{\text {reflected }}$, where $\theta_{\text {incident }}=10^{\circ}$. For diffuse measurements the sample is placed such that $\theta_{\text {reflected }}=\theta_{\text {incident }}+18^{\circ}$, further discussed in the next section. 


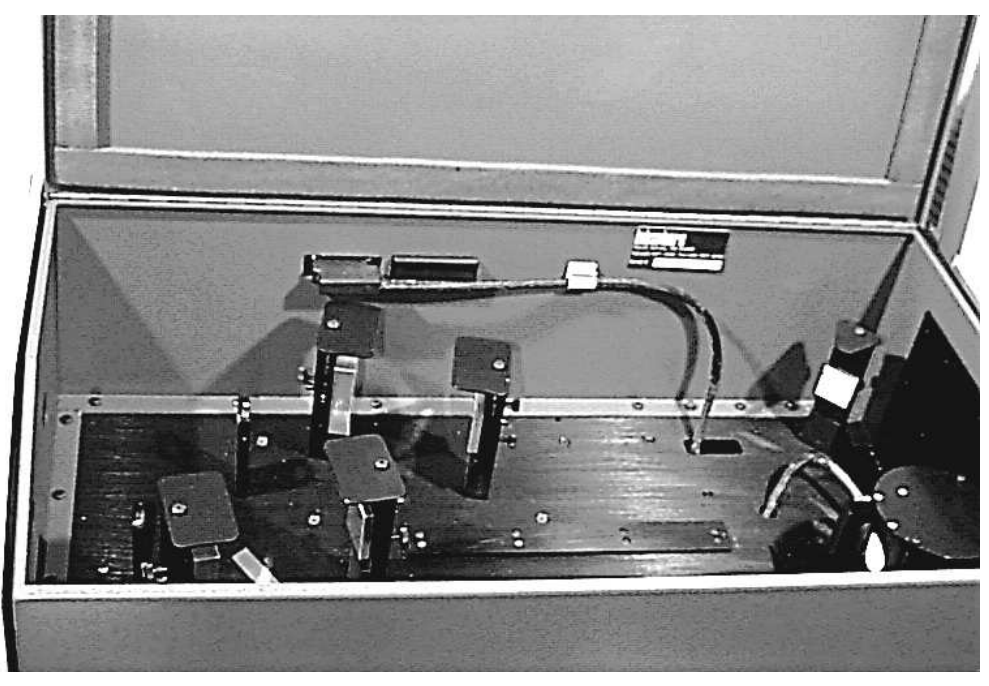

Figure 7: Experiment Setup(Drawing) (Perk 6 Elmer -18 spectrometer)

Lambda-18 Light Box

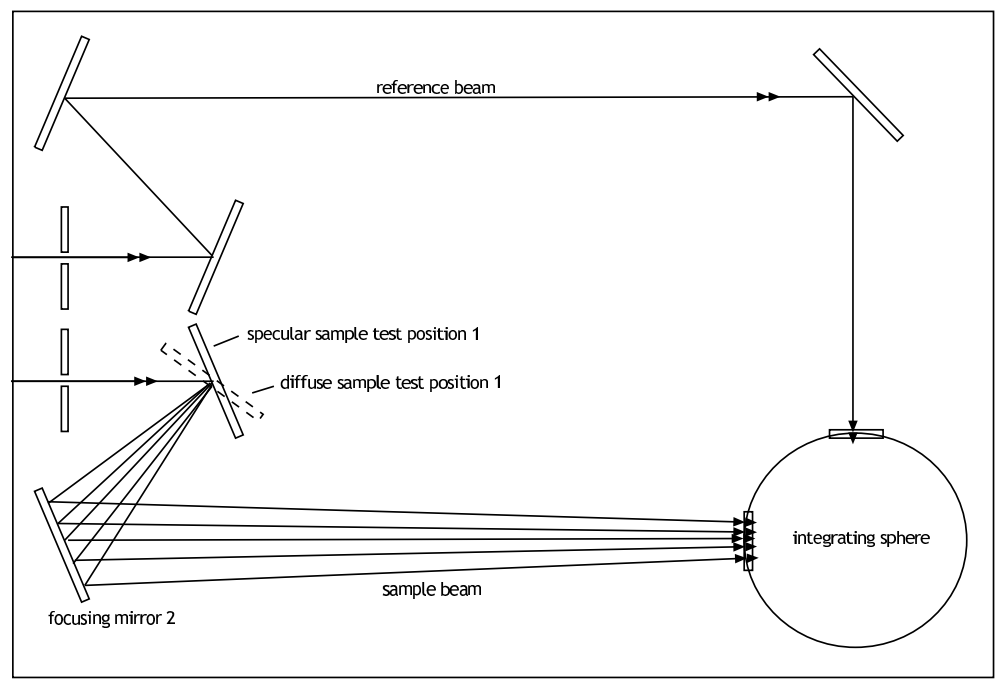

Figure 8: Experiment Setup(Drawing) (Perk 85 Elmer -18 spectrometer)

\subsection{Measurement Technique}

Within the Perk \& Elmer Lambda 18 reflectance spectrometer a monochromator light source is used in a dual-beam arrangement. The beams are 
chopped at $1 \mu$ s intervals into a reference (ref) beam and a sample (sam) beam. This eliminates systematic errors due to PMT drift. Each beam is directed into an integrating sphere (IS) where a PMT measures the light input $L_{r e f}$ and $L_{s a m}$ as the monochromator scans. The actual resulting measurement is the ratio $L_{\text {sam }} / L_{r e f}$.

The TYVEK samples $\left(3 \times 3 \mathrm{~cm}^{2}\right.$ active area) were placed flat in a sample holder near mirror 1 in the sample beam line. Part of the light $(\epsilon)$ is reflected into focusing mirror 2 and then into the integrating sphere. The beam angle of incidence with respect to the sample holder is normally at $10^{\circ}$ for optimizing specular reflectance measurements. This angle was then increased to $28^{\circ}$ for diffuse scattering measurements. The diffusely scattered light will produce the dominate source of photoelectrons to finally reach the Auger tank photomultiplier tubes. This simple two-angle approach was taken to avoid serious modifications to the spectrometer that would be necessary to sweep the angle. The monochromator was set to scan between $\lambda=200 \mathrm{~nm}$ and $\lambda=600 \mathrm{~nm}$.

A measurement is taken by first running a blank sample $(A)$ for baseline correction and then a second run by inserting the TYVEK $(B)$ sample. After the second TYVEK scan a baseline corrected spectrum ratio (S) is calculated,

$$
S(\lambda, \Delta \theta)=\frac{B(\lambda, \Delta \theta)}{A(\lambda)}
$$

, where $\Delta \theta$ indicates that a fraction $\epsilon(\lambda)$ of light is collected in the $B$ scan only if it scatters within the solid angle coverage of mirror 2 and into the 
integrating sphere. All incident light is collected in the normalizing $A$ measurement. Thus the true reflectivity is

$$
R(\lambda)=S(\lambda, \Delta \theta) / \epsilon(\lambda)
$$

We do not determine $\epsilon(\lambda)$ but only report the spectrum ratios $S(\lambda)$.

\subsection{Test Scans}

There were 12 kinds of Tyvek samples that have been tested. A specular and diffuse scan was taken of each as a function of wavelength. In addition each group of scan was accompanied by a scan of a $S T A N D A R D$ sample later used in correcting scans taken on different days. This STANDARD sample is denoted as sample $1 A-1025 D$.

The results of the Tyvek scans are shown in Figures 9, 10, and 11. Here the spectrum ratios are shown in pairs of two scans, a specular(top) and diffuse(bottom). The ordinate of each graph is given in arbitrary units. The integrated sum is given below each scan in both an unweighted (SUM) and weighted (WSUM) version. The first sum is the unadjusted sum. The second sum involves a weighted sum.

The photoelectron yield reaching the the PMTs will be a convolution of incident radiation spectrum $d N / d \lambda$, transmission $\mathrm{T}(\lambda)$, PMT response $\mathrm{Q}\left(\lambda, \theta_{\text {tube }}\right)$, and reflectivity $R^{n}\left(\lambda, \theta_{\text {tyvek }}\right)$. For simple comparisons,'s we assume $\mathrm{n}=1$ bounce, and transmission $\mathrm{T}=1$. We perform an integral of the form,

$$
W S U M=K \int_{300 n m}^{500 n m} Q(\lambda) R(\lambda) d \lambda / \lambda^{2}
$$



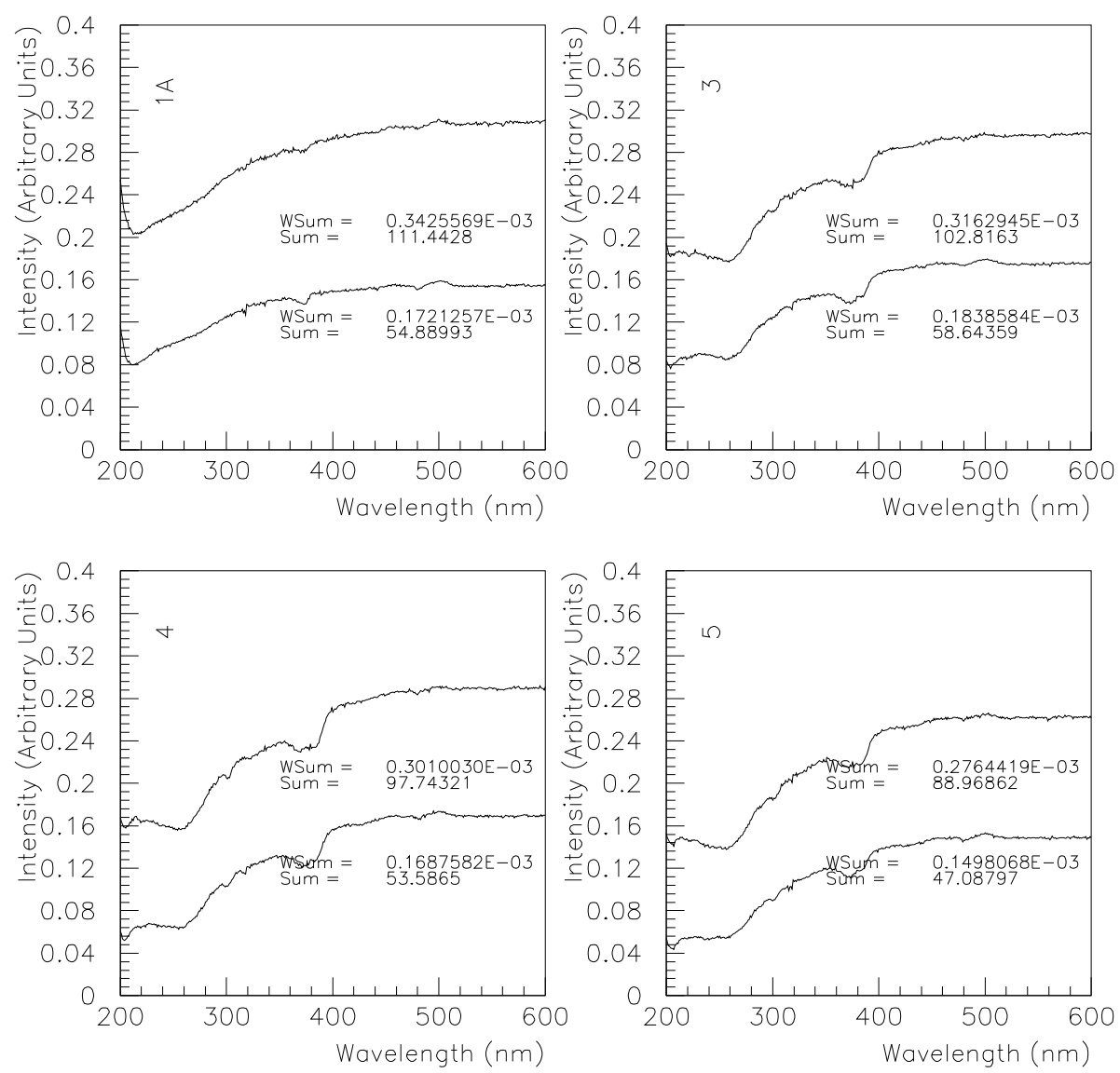

Figure 9: The Reflectivity Tests 

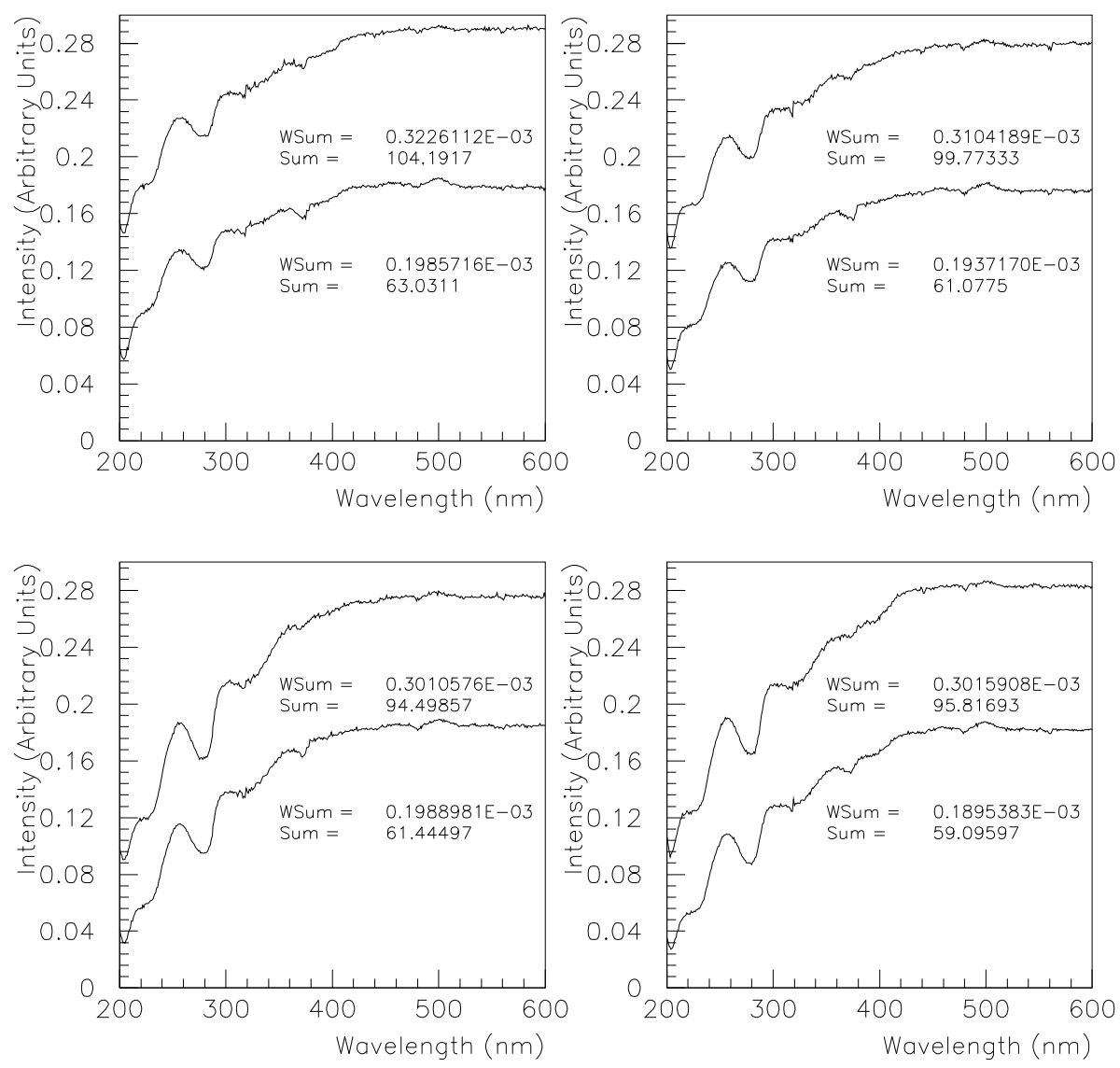

Figure 10: The Reflectivity Tests 

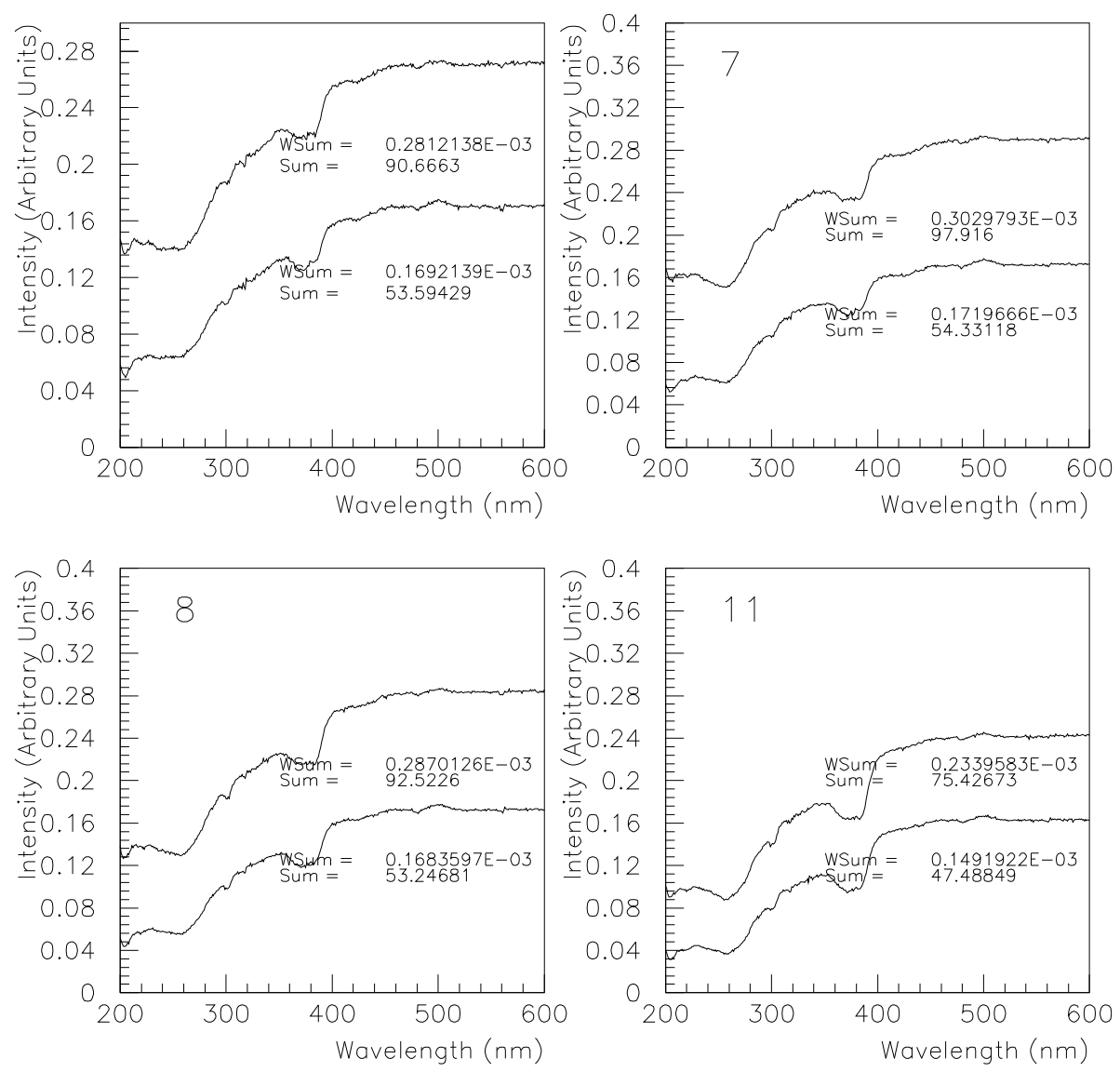

Figure 11: The Reflectivity Tests 
Thus we adjust the data before summing it by multiplying each data point by the photodetector quantum efficiency multiplied by the characteristic $1 / \lambda^{2}$ for Cerenkov radiation. $K$ is the arbitrary scale factor common to all measurements. We believe that WSUM is a better figure of merit with which to judge the net effect of the reflectivity for the Tyvek samples. These measurements are summarized in Table 2.

Table 2: Integral $(W S U M)$ values $\left(10^{-3}\right)$ of different Tyvek materials for Specular and diffuse reflection using Perk and Elmer Lambda-18 Spectrometer.

\begin{tabular}{|c|c|c|c|c|}
\hline Tyvek Type & Specular & Diffuse & Specular corrected & Diffuse corrected \\
\hline 1A 1025D & 0.3426 & 0.1721 & 0.3276 & 0.1723 \\
\hline 3 1025D & 0.3163 & 0.1839 & 0.3054 & 0.1962 \\
\hline 4 1056D & 0.3010 & 0.1688 & 0.2766 & 0.1654 \\
\hline 5 1058D & 0.2764 & 0.1498 & 0.2331 & 0.1302 \\
\hline 6 1059B & 0.2812 & 0.1692 & 0.2414 & 0.1662 \\
\hline 7 1059D & 0.3030 & 0.1720 & 0.2802 & 0.1717 \\
\hline 8 1070D & 0.2870 & 0.1684 & 0.2452 & 0.1646 \\
\hline $91073 \mathrm{~B}$ & 0.2765 & 0.1599 & 0.2765 & 0.1599 \\
\hline $101073 \mathrm{D}$ & 0.2707 & 0.1677 & 0.2707 & 0.1677 \\
\hline 111079 & 0.2340 & 0.1492 & 0.1671 & 0.1292 \\
\hline $121085 \mathrm{D}$ & 0.2801 & 0.1611 & 0.2395 & 0.1506 \\
\hline Sunluk I & 0.3045 & 0.0859 & 0.2830 & 0.04102 \\
\hline Sunluk 8k & 0.1533 & 0.1015 & 0.0717 & 0.0598 \\
\hline SR-1 & 0.3072 & 0.1945 & 0.2881 & 0.1580 \\
\hline SR-2 & 0.2931 & 0.2035 & 0.2606 & 0.1653 \\
\hline SR-3 & 0.2983 & 0.1866 & 0.3276 & 0.1515 \\
\hline SR-4 & 0.2462 & 0.1818 & 0.3276 & 0.1476 \\
\hline
\end{tabular}




\subsection{Corrections to the Data}

Since a $S T A N D A R D$ Tyvek sample scan has been taken with each operation of the spectrometer. We can also make a normalizing correction to each sample scan to account for scans taken on different days. All STANDARD scans were scaled to the first such scan. Again the integral WSUM for the diffuse and specular reflection is found by summing the numbers as previously discussed. Data from the standard 1A-1025D sample taken at the beginning of each measurement set is used to correct the the data for that set by renormalizing all measurements to the measurements of the first day of running.

By comparing the later $S T A N D A R D$ runs to the first day tests, a scale factor is determined and applied to all data in the current set. A summary of WSUM values, both corrected and non-corrected are shown in Table 1 for comparisons,

$$
W S U M_{C O R R}=W S U M \times W S U M_{S T A N D A R D 0} / W S U M_{S T A N D A R D}
$$

\subsection{Error Analysis}

As mentioned before we expected a natural fluctuation of our measurements due to spectrometer measurement error, measurement technique, and variation of sample.

From repeated measurements of the same samples (not moved) we determined that the Perk\&Elmer Lambda-18 spectrometer provided reproducible 
measurements of the reflectivities at the $\frac{1}{3} \%$ level, confirming spectrometer literature reports.

A study of error due to sample placement technique was performed by repeating scans on a given sample 2 to 3 times after removal and replacement. A $1.5 \%(1 \sigma)$ error measurement technique was established.

To estimate errors in sample variation, we performed repeated measurements of 4 Tyvek samples cut from the same roll (TYVEK 1025D) but from 4 random positions. Error due to sample variation was established from measurements of the 4 TYVEK 1025D samples cut from random locations and assigned an $7 \%$. error.

Thus the total measurement error on the Tyvek samples when taken in quadrature

$$
\sigma_{t o t}(\%)=\sqrt{(.3 \%)^{2}+(1.5 \%)^{2}+(7.0 \%)^{2}}
$$

resulting in a $\sigma_{t o t}(\%)=8 \%$ error, dominated by sample variation. From this error we might expect $(10-15) \%$ shifts in our spectrum analyses. The measurements and errors are shown in Figure 12. 


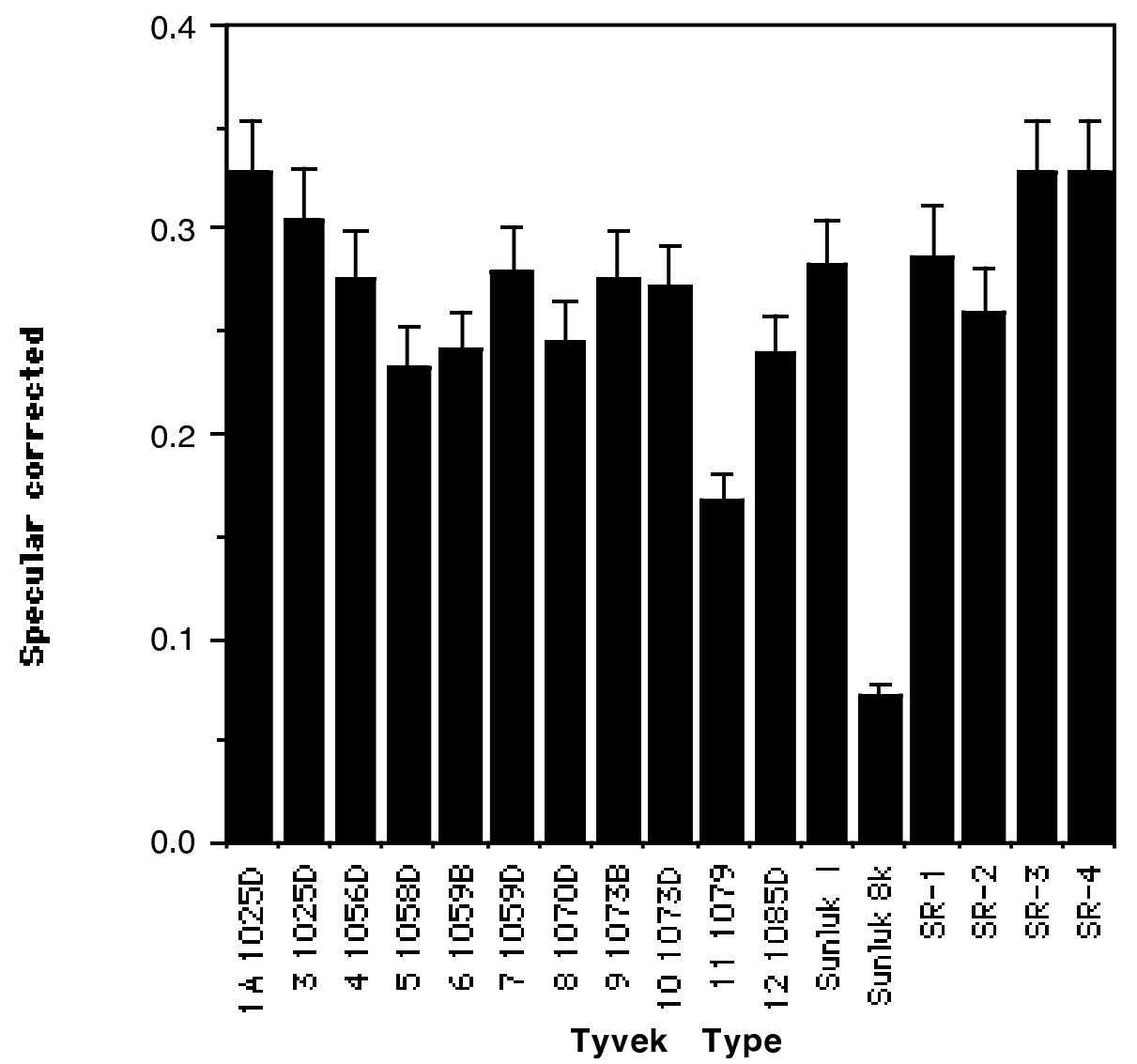

Figure 12: Corrected Specular \& Diffuse Histograph for the 12 Tyvek samples with $8 \%$ level error

\section{TYVEK Reflectivity versus Incident and Reflected Angles}

\subsection{Theory}

Light reflected from a given surface depends on several factors (1) Type of the surface e.g quantity of light reflected from a polished silver surface is much greater than that reflected from polished steel. (2)The color of the surface. 
(3) The angle at which the incident light meets the reflecting surface.

The reflected light is usually characterized by two components, diffuse and specular which is also the case for the DUPONT tyvek material which we have done the study on. Therefore the first component produced by diffuse character of Tyvek can be described by a function form

$$
I_{d} \theta_{r}=I_{d} \cos \left(\theta_{r}\right)
$$

this is the so called Lambert's cosine law [18].

Lambert's law states that the reflected energy from a small surface area in a particular direction is proportional to cosine of the angle between that direction and the surface normal. Lambert's law determines how much of the incoming light energy is reflected. The intensity depends on the light source's orientation relative to the surface, and it is this property that is governed by Lambert's law.

The second component is produced by specular character of Tyvek. Specular reflection (regular reflection) occurs when incident parallel rays are also reflected parallel from a smooth surface. These second component can be described assuming the gaussian peak with an intensity varying with $\theta_{i}$ and takes the form

$$
I_{F} \theta_{r}, \theta_{i}=I_{F} \theta_{i} \exp \left(-\theta_{r}-\theta_{i}\right)^{2} /\left(2 \sigma^{2}\right)
$$


Therefore the total intensity at the reflection angle $\theta_{r}$ is given by :

$$
l\left(\theta_{r}, \theta_{i}\right)=l_{d}\left(\theta_{r}\right)+l_{F}\left(\theta_{r}, \theta_{i}\right)
$$

which is characterized by both diffuse and specular components.

\subsection{Experimental Setup and Procedure}

A standard He-Ne laser beam $(\lambda=640 \mathrm{~nm}), 3.05$ optical fiber and intensity photometer(PASCO OS-8020) were used for the measurements. The HeNe laser beam was used to illuminated a thin sample of Tyvek material which was located about $12 \mathrm{~cm}$ from the source on a holder, see Fig 13. To measure the amount of reflected light at several angles we have used a $3.05 \mathrm{~mm}$ diameter optical fiber, optically coupled to a relative intensity photometer. The sample holder and optical fiber were allowed to rotate on a goniometer of $8.5 \mathrm{~cm}$ in radius while the incidence laser beam was impinging on the sample from a fixed direction.

By switching between different scale sensitivities on the photometer we were able to measure the relative intensity of the reflected beam at the reflected plane. We took two kind of data at every angle we used. We first took data the laser turned off and used it as a background substraction for the second measurement we took with the laser on. No absolute measurements

of the light intensity was obtained, since we were not able to measure the small light output coming out of the optical fiber. 


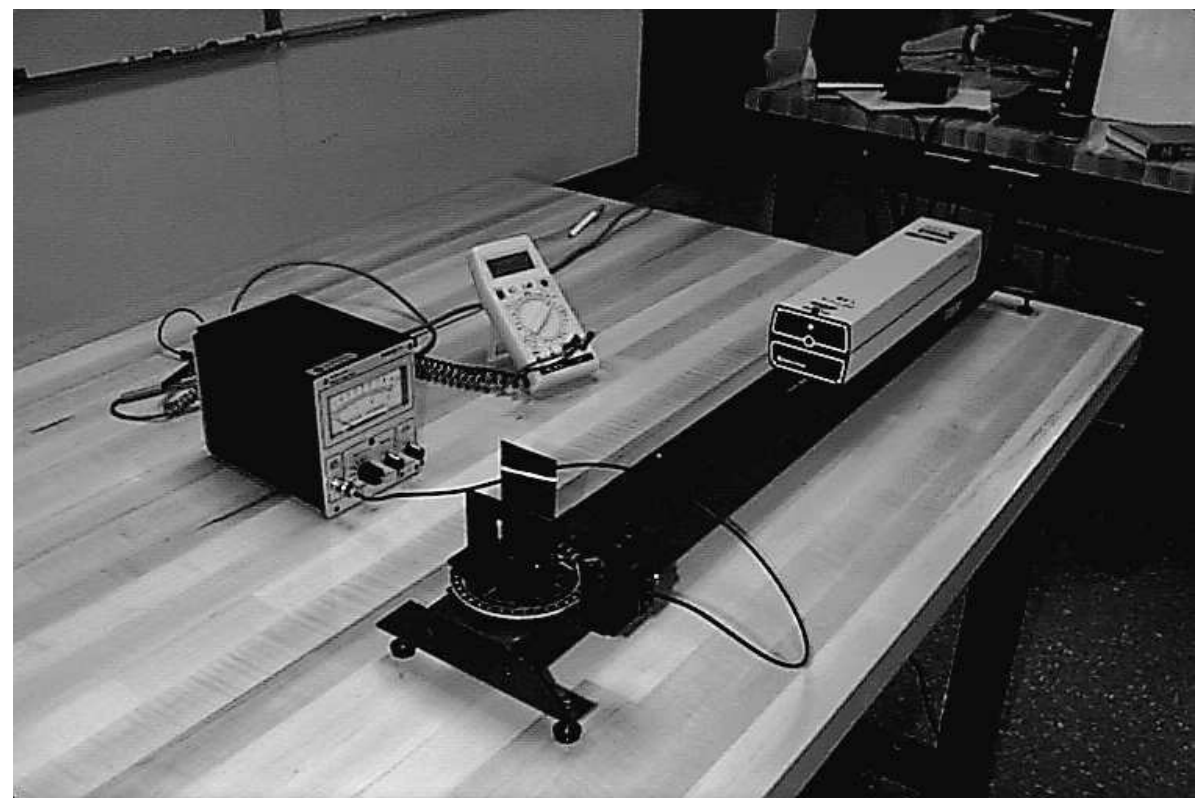

Figure 13: The Experiment Setup

\subsection{In-the-Plane Measurements}

We have taken three measurements at incident angles $0^{\circ}, 30^{\circ}$ and $45^{\circ}$ respectively, We have used PAW [19] software to generate the figures shown Fig. 14 and Fig. 15 where the light intensity as a function of the angle, $\theta_{r}$ is plotted. The relative intensity is given in arbitrary units. By using a fitting function routine

REAL FUNCTION TYVEK10(X)

COMMON $/$ PAWPAR/PAR $(3) X X=0.0175 \times \theta_{r}$

$X X X=0.0175 *\left(\theta_{r}-\theta_{i}.\right)$

$S I G=0.0175 * P A R(3)$ 
$T Y V E K 10=P A R(1) * C O S(X X)+P A R(2) * E X P(-.5 *((X X X) / S I G) * * 2)$

\section{RETURN}

\section{END}

we are able to fit the graphs. The values $\operatorname{PAR}(1)$ and $\operatorname{PAR}(2)$ and $\operatorname{PAR}(3)$ are generated by PAW fitting function. Our fitting function corresponds to the characteristic function form of specular and diffuse components. The first first component is produced by diffuse character of Tyvek. By using the Lambert's cosine to fit our data without the peak, we found that diffuse component is independent of $\theta_{i}$ since the average amplitude I obtained is essentially the same for incident angles. We can evaluate its relative contribution by evaluating the Integral

$$
I=A \int_{-\pi / 2}^{\pi / 2} \cos \left(\theta_{r}\right) d \theta_{r}
$$

The second component is produced by the specular character of Tyvek and it's relative contribution is determined by evaluating the integral

$$
I\left(\theta_{r}\right)=B \int_{-\pi / 2}^{\pi / 2} \frac{e^{\left(\theta_{r}-\theta_{i}\right)^{2}}}{2 \sigma^{2}} d \theta_{r}
$$

where $\mathrm{A}=\operatorname{PAR}(1)$ and $\mathrm{B}=\operatorname{PAR}(2)$. The results are shown in table 3 .

From our fit we found that $\sigma$ to be a constant of value $\sigma=22^{\circ}$ for all incident angle. From the above results the reflected intensity can be understood as the reflection of light in the reflection plane by a dielectric material. The total intensity of light at the reflection angle $\theta_{r}$ is then $I\left(\theta_{r}, \theta_{i}\right)=I_{d}\left(\theta_{r}\right)+I_{F}\left(\theta_{r}, \theta_{i}\right)$ This quite simple model agrees with our data 
INPLANE SCAN at O DEGREES

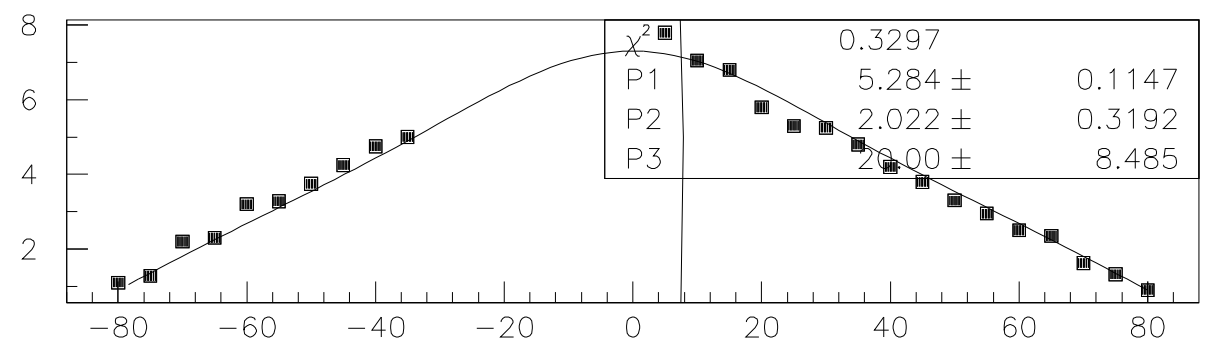

INPLANE SCAN at 10 DEGREES
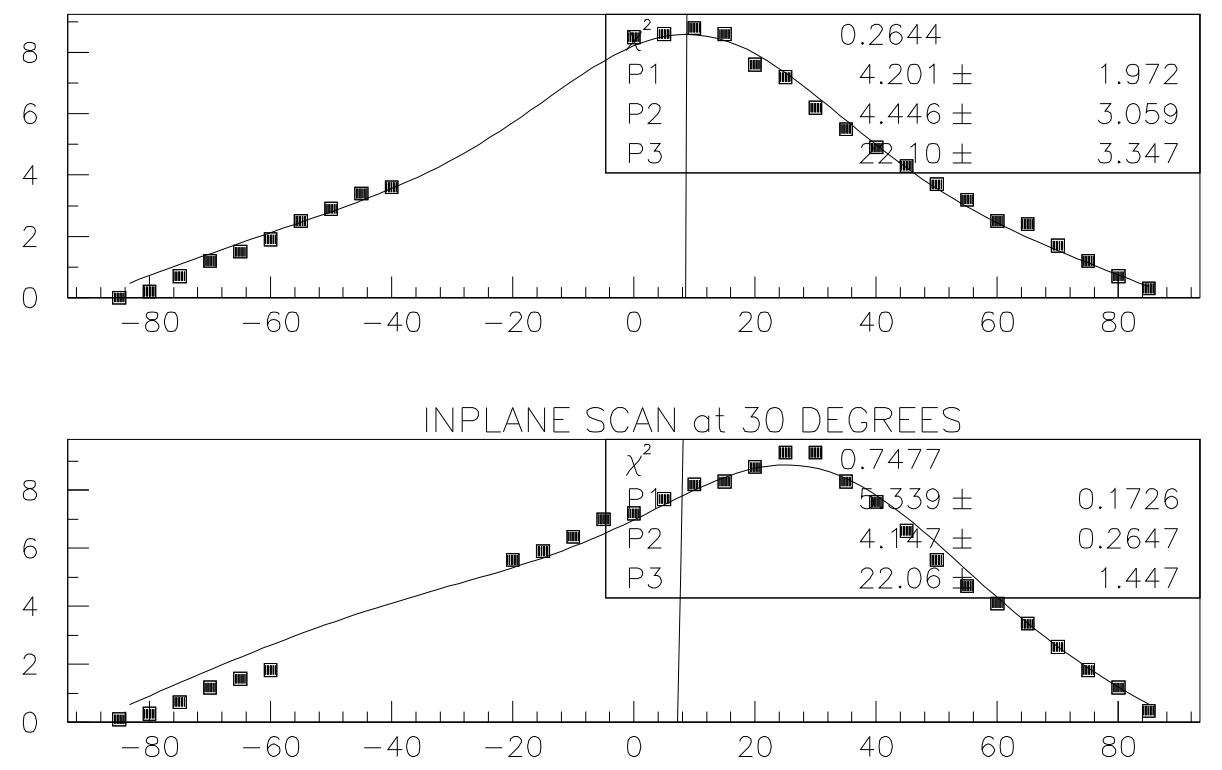

Figure 14: Intensity of the reflected light in arbitrary units as a function of the reflected angle for incident angles $0^{\circ}, 10^{\circ}$, and $30^{\circ}$

Table 3: Integral values $\left(10^{-3}\right)$ of different measurements for different incidence using PASCO photometer and laser.

\begin{tabular}{|c|c|c|c|c|c|}
\hline$\theta_{i}$ & $\mathrm{~A}$ & $\mathrm{~B}$ & Lambert cosine & Gaussian Peak & Total \\
\hline $0^{\circ}$ & 5.284 & 2.022 & 10.568 & 79.866 & 90.434 \\
\hline $10^{\circ}$ & 5.201 & 4.446 & 10.234 & 161.37 & 171.604 \\
\hline $30^{\circ}$ & 5.339 & 4.147 & 10.678 & 340.343 & 351.02 \\
\hline
\end{tabular}


OUT-OF-PLANE SCAN at O DEGREES

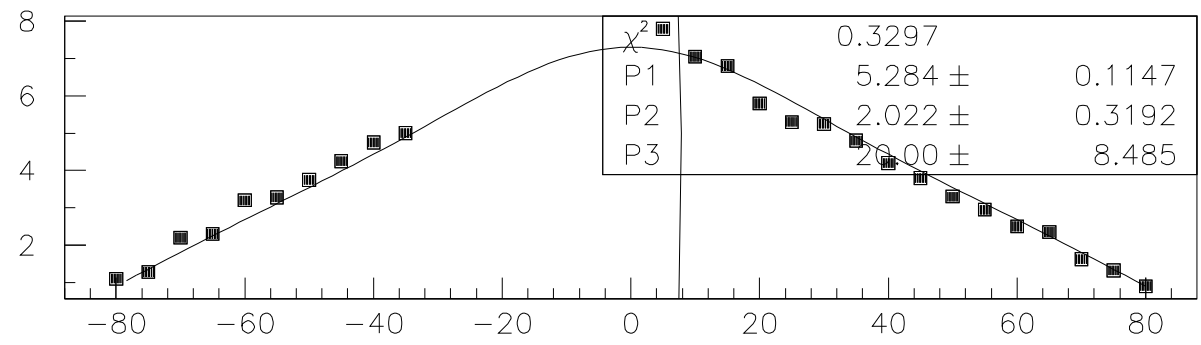

OUT OF PLANE SCAN at 35 DEGREES



OUT OF PLANE SCAN at 45 DEGREES



Figure 15: The Reflectivity Tests 
Table 4: Integral values $\left(10^{-3}\right)$ of different measurements for different Out of plane angles using PASCO photometer and laser.

\begin{tabular}{|c|c|c|c|c|}
\hline Incidence angle & $\mathrm{A}$ & $\mathrm{B}$ & Lambert cosine & Gaussian Peak \\
\hline $0^{\circ}$ & 5.284 & 2.022 & 10.568 & 79.866 \\
\hline $35^{0}$ & 6.022 & 2.88 & 12.044 & 47.26 \\
\hline $45^{\circ}$ & 6.577 & 1.108 & 13.154 & 169.58 \\
\hline
\end{tabular}

quite well.

\subsection{Out-of-Plane Measurements}

We conducted measurements at three different angles 0, 30, 45 degrees out of the plane of normal reflection. The setup still was the same as the previous experiment except that we had the Tyvek material placed at the three outof-plane angles. We expected diffuse scattering to dominate. The results are shown in Table 4 and the Figure 15. As expected the diffuse scattering is seen to dominate in all cases. 


\section{Tank Simulation}

I have used a Monte Carlo program 'TANK'[20] which simulates the passage of particles through the Auger water cerenkov tanks. In the simulation the tank is given a height of $1.2 \mathrm{~m}$ and a radius of $1.8 \mathrm{~m}$ and is filled with water of index of refraction $n=1.33$ and mean absorption length as a function wavelength of $\lambda_{a b s}=7 \mathrm{~m}$. The walls of the tank are lined with TYVEK and three PMTs are place to collect light at the tank surface.

Electron at ground level from air showers are typically of energy E=100 $\mathrm{MeV}$ and muons have a mean energy of $1000 \mathrm{Mev}$. The electron travels about $20 \mathrm{~cm}$ in the water tank before loosing all of its energy. It will radiate this energy away partially as ionization excitation and cerenkov light in a nearly random and isotropic manner. $1000 \mathrm{MeV}$ muons will pass through the $1.2 \mathrm{~m}$ high tank nearly undeflected loosing about $250 \mathrm{MeV}$ along the way. Muons will produce cerenkov radiation along the straight line trajectory of cerenkov angle $\cos (\theta)=1 / 1.33$. These processes are simulated 'TANK'

The scattering of light from the TYVEK walls in TANK is controlled by the user and we have used the parameterization determined in my tests for this purpose. we have assumed that $70 \%$ of the time the scattering is of the random type and $30 \%$ of the time it is of the gaussian type with half-angle 15 degrees, Figure 16(a). For these studies the TYVEK reflectivity is taken from our spectrophotometric measurements and parameterized as $90 \%$ from $600 \mathrm{~nm}$ to $400 \mathrm{~nm}$ and then linearly dropping to $60 \%$ at $200 \mathrm{~nm}$ wavelength, 


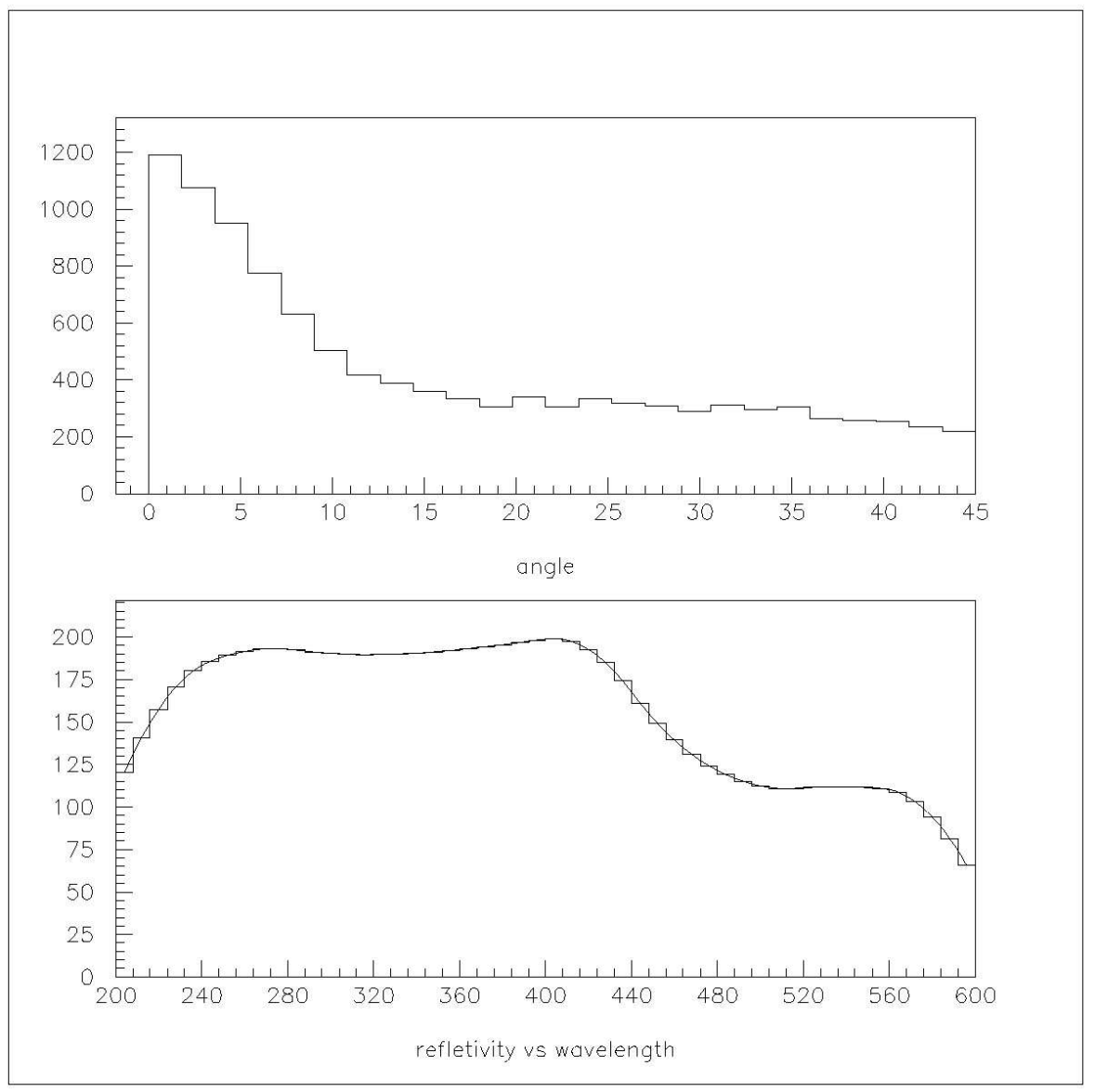

Figure 16: Reflectivity dependence of the wavelength and the Incident angle

Figure 16(b). The time structure of the light arriving at the PMTs is also kept so time of arrival of photons can be studied.

I use the simulation to determine the number of photons collected by the PMT's for electrons and muons and the time of arrival of these signals for various trajectories. It has been suggested that blackening the top of the surface array tanks will decrease the average time of arrival of photons with some lose in number. I looked at this issue with TANK. In some cases I 


\begin{tabular}{|c|c|c|c|c|}
\hline Type & No. of photons(e) & Time(e)(ns) & No. of Photons $(\mu)$ & Time $(\mu)(\mathrm{ns})$ \\
\hline$A_{\text {white }_{\text {op }}}$ & 14 & 50.6 & 25 & 23.5 \\
\hline$A_{\text {black }_{\text {op }}}$ & 7 & 22.1 & 14 & 15.9 \\
\hline$B_{\text {white }_{\text {op }}}$ & 18 & 50.2 & 18 & 33.0 \\
\hline$B_{\text {black }_{\text {op }}}$ & 6 & 20.3 & 14 & 21.4 \\
\hline$C_{\text {white }_{\text {op }}}$ & 18 & 33.7 & 22 & 42.5 \\
\hline$C_{\text {black }_{\text {op }}}$ & 5 & 25.9 & 9 & 28.3 \\
\hline
\end{tabular}

Table 5: Number of Cerenkov photons collected per thousand and their mean time of arrival.

can compare my results to actual measurements performed by Pierre Auger collaboration.

\subsection{Measurements}

We choose to explore three typical trajectories $\mathrm{A}, \mathrm{B}$, and $\mathrm{C}$ of the electrons and muons entering the tank from above. These are defined below.

$$
\begin{aligned}
& A=\operatorname{xin}=0 . \text { yin }=0 . \text { xout }=0 . \text { yout }=0 . \\
& B=\operatorname{xin}=0 . \text { yin }=0 . \text { xout }=1 . \text { yout }=1 . \\
& C=\operatorname{xin}=1 . \text { yin }=1 . \text { xout }=0 . \text { yout }=0 .
\end{aligned}
$$

We have performed simulations both for white top and black top for each trajectories and recorded the number of photons collected and time of arrival of these signals for electrons and muons. A comparison between photon yields and timing is shown in Table 5 .

We can use the simulation to estimate the mean number of photoelectrons detected for vertical muons by noting that approximately 46000 photons 


\begin{tabular}{|c|c|c|}
\hline Type & $\frac{B T}{W T}$ (electrons) & $\frac{B T}{W T}$ (muons) \\
\hline A & 0.436 & 0.675 \\
\hline B & 0.405 & 0.646 \\
\hline C & 0.771 & 0.667 \\
\hline
\end{tabular}

Table 6: Ratio of the Mean time of arrival for black top and white top. are produced as shown earlier. The efficiency for detecting these is $\epsilon_{\text {det }}=$ $25 / 1000=.025$. Using a quantum efficiency $\mathrm{Q}=.15$ we obtain the average number of photoelectrons collected is

$$
N_{\text {p.e. }}=46000 \times \epsilon_{\text {det }} \times Q=172,
$$

a value close to that observed in data.

In Table 6 we have found the ratios of mean signal collection times for muons and electrons $\left(\frac{\text { black }_{t} o p}{\text { whitet }_{\text {op }}}\right)$ using vertical trajectory A. From the table its shown that the muons arrival time decreases about $\approx 67 \%$ and the electrons arrival time decreases $\approx 43 \%$ for the central vertical going-through muons and electrons trajectories in average when using a black top. In Table 7 we calculate the ratio of collected signal. We see that number of photons collected decreases about $\approx 56 \%$ for muons and $\approx 50 \%$ for electrons when using the black top.

We can compare these results with those of a prototype water Cerenkov detector running at Tandar.[20] In Tandar experiment they have examined the possibility of reducing the long decay time constants measured by water tank detectors by using a black top surface instead of a fully lined Tyvek tank. 


\begin{tabular}{|c|c|c|}
\hline Type & $\frac{B T}{W T}$ (electrons) & $\frac{B T}{W T}$ (muons) \\
\hline A & 0.50 & 0.56 \\
\hline B & 0.33 & 0.78 \\
\hline C & 0.28 & .41 \\
\hline
\end{tabular}

Table 7: Ratio of the number of photons collected for black top and white top.

In their experiment they found that the use of black top surface effectively reduces the pulse duration from $\approx 40 \mathrm{~ns}$ to $\approx 10 \mathrm{~ns}$ at the expense of a $60 \%$ decrease in the total charge collected. Fig 17 shows the results of the average pulse measured by one of the photomultiplier for a muon trajectory(which in our case is trajectory $A$ ) where it can be seen a dramatic shortening of the decay constant from 39 ns to $11 \mathrm{~ns}(\approx 70 \%)$ is evident. These results are consistent with our calculations. 




Figure 17: Comparison of average pulses due to central vertical through-going muons with tyvek top and black lining on the top. 


\section{Conclusions}

From the studies performed in the thesis one concludes that there is no great difference in the reflectivities of TYVEK samples in the wavelength range $\lambda$ $=(300-700) \mathrm{nm}$. The diffuse reflectivities of $1025 \mathrm{D}$ and $1073 \mathrm{D}$ are similar. This other quality factors of these sample should be used in determining the bag liner. These tests were able to clearly reject for example the SANLUK samples obtained from Brazil and thus could make a reasonable quality control test for bag material used in fabrication.

Measurements of the reflectivity versus incident and reflected angle is closely matched by a simple gaussian plus random scatter model. The out-ofplane measurements are the first to be performed and also show no surprises fitting the random scatter model well.

After inserting experimental information into our TANK simulation program we were able to make good predictions for the number of photoelectrons, time structure of the signal, and differences in detector design (eg. black top vs. white top). These predictions are in good agreement with present Pierre Auger water-Cerenkov test measurements on cosmic rays. The TANK simulation may ultimately lead to other tank design optimizations. 


\section{References}

[1] J. Linsley, Phys. Rev. Lett 10, 146 (1993).

[2] D. J. Bird et al., Astrophys. J. 441, 144 (1995).

[3] H. Hayashida et al., Phys. Rev. lett. 73, 3491 (1994).

[4] Tyvek diffuse reflectivity. F. Hasenbalg \& D. Ravignani, GAP 97-035

[5] Pierre Auger design report

[6] Consultant's report. SOC-R950-195 Surface optics corp. san Diego, CA (for U.C - Irvine, 1995)

[7] Introduction to Elementary Particles, David Griffiths , 1987

[8] Physicals Review D Particles 6 Fields, III.24 1992

[9] M. A. Lawrence, R. J. O. Reid and A. A. Watson,J. Phys. G17, 733 (1991)

[10] Measuring the Highest Energy Cosmic Rays: The Auger Project, J. Matthews, GAP-97-007

[11] Glenn S. Smith, Classical Electromagnetic Radiation (1997)

[12] Physical Review D Particles and Fields Volume 45 NO. 11 (1992) 
[13] The current status of the Super-Kamionde experiment, K. Martens Talk given at the 25th International INS symposium, Tokyo Dec 1996 http://www-sk.icrr.u-tokyo.ac.jp/doc/sk/pub/

[14] Milagro Physics and Detector Construction status, mei-li chen Fo8.11 http://flux.aps.org/meetings/BAPSAPRR95/abs/spo811.html

[15] D.J. Bird et al. Astrophys. J. 424, 491 (1994); R. Baltrusaitis et al. , Nucl. Instrum. Methods A240, 410 (1985)

[16] D.J. Bird et al., Proc. 23d ICRC(Calgary) 2, 450 (1993).

[17] Directional Reflectance Measurements(DR) on Two special sample Materials-Final Report., SOC-R950-001-0195, Prepared for the University of California, Irvine, School of Physical Sciences Irvine, California 92717-4675, January 1995.

[18] Principles of Optics, M. Born and E. Wolf, Pergammon Press, Oxford, England(1980)

[19] CERN Physics Analysis Workstation software, CERN Computer Center Program Library.

[20] Program developed by J. Gichaba and L. Cremaldi at the University of Mississippi. 
[21] Experimental Comparison in Water Tank Detectors With Tyvek and Black Top Surfaces, F. Hasenbalg, P. Bauleo, A. Ferrero, A. Filevich, C. Guerard, D.Ravigani, J. Martino, GAP-97-027 